\title{
Cluster multipole dynamics in noncollinear antiferromagnets
}

\author{
Takuya Nomoto $\oplus^{1, *}$ and Ryotaro Arita $\oplus^{1,2}$ \\ ${ }^{1}$ Department of Applied Physics, The University of Tokyo, Hongo, Bunkyo-ku, Tokyo 113-8656, Japan \\ ${ }^{2}$ RIKEN Center for Emergent Matter Science (CEMS), Wako 351-0198, Japan
}

(Received 11 March 2019; accepted 3 February 2020; published 25 February 2020)

\begin{abstract}
A systematic framework to investigate the spin dynamics in a noncollinear antiferromagnet is proposed. Taking $\mathrm{Mn}_{3} \mathrm{Sn}$ as a representative example, we derive an effective low-energy model based on the multipole expansion of the magnetic structure, and investigate the uniform precession and the domain wall dynamics. We show that the solution for the effective model accurately reproduces the numerical calculation of the Landau-Lifshitz-Gilbert equations. Our results indicate that $\mathrm{Mn}_{3} \mathrm{Sn}$ has preferable properties for applications to a racetrack memory and a spin torque oscillator, and thus is a promising candidate for spintronics devices by using the multipole degrees of freedom.
\end{abstract}

DOI: 10.1103/PhysRevResearch.2.012045

Introduction. In the field of spintronics, spin manipulation based on an antiferromagnet (AFM) has attracted much attention because of its potential advantages over a ferromagnet (FM) [1-7]. For example, due to the absence of net magnetization, AFM devices are relieved of the stray field problem, which is one of the main obstacles to high-density integration. A maximum velocity of a domain wall induced by a spin current, thermal gradient, and staggered field is much faster in collinear AFM than in FM [8-11], which is a favorable property for applications to racetrack memories. A typical energy scale of AFM is also much higher than that of FM, resulting in a fast switching of its magnetization $[12,13]$ as well as a coherent precession with the $\mathrm{THz}$ frequency [14-17]. The ac signals generated by such steady motion can be extracted as the ac voltage through inverse spin-Hall effects or as dipolar radiation in a special case $[18,19]$.

Despite such fascinating properties, however, so far there have been few realizations of AFM devices. This is mainly because the Néel vector, the order parameter of collinear AFM, does not couple directly to the external field. Since collinear AFM usually possesses time-reversal symmetry, it does not show any directional signal associated with symmetry breaking such as the anomalous Hall effect and magnetooptical Kerr effect. For example, in a racetrack memory, it is necessary to detect each domain separated by the domain walls, but it is impossible in conventional collinear AFM. One possibility to overcome the problem is to use a ferrimagnet [20-25]. Although it has features of both FM and AFM, a usual ferrimagnet shows a fast response only near its compensation point.

In this Rapid Communication, we focus on another possibility of AFM, namely, noncollinear AFM. Recently, it

*nomoto@ap.t.u-tokyo.ac.jp

Published by the American Physical Society under the terms of the Creative Commons Attribution 4.0 International license. Further distribution of this work must maintain attribution to the author(s) and the published article's title, journal citation, and DOI. was shown that the Weyl AFM $\mathrm{Mn}_{3}$ Sn [26] has a tiny net magnetization about $2 \mathrm{~m} \mu_{B}$ /atom but shows large anomalous Hall and Nernst effects comparable to a conventional FM [27-32]. The spin texture in its Néel state is regarded as a ferroic order of a cluster octupole whose symmetry is the same as a conventional dipole under hexagonal point group symmetry [33]. The related AFM $\mathrm{Mn}_{3} \mathrm{Ge}$ also shows a large anomalous Hall effect and has a noncollinear spin texture [30,34]. Thus, one may expect that noncollinear AFM is a promising platform for magnetic devices since it is AFM and its spin dynamics is detectable by the same methods as FM.

In contrast to FM and collinear AFM, theoretical studies on the spin dynamics of noncollinear AFM are limited [35-38]. Especially, there is a lack of systematic methods to obtain its effective model so far. Here, we propose a framework to derive an effective model of noncollinear AFM based on the cluster multipole theory $[33,39,40]$. $\mathrm{In} \mathrm{Mn}_{3} \mathrm{Sn}$, the derived model is composed of two octupole degrees of freedom and reduced into the sine-Gordon model similar to FM and collinear AFM. We check the validity of the model by comparing two phenomena to these in the original model: the domain wall dynamics and steady-state precession. The agreement is very good at low energy, which means that the spin dynamics in $\mathrm{Mn}_{3} \mathrm{Sn}$ is almost dominated by the octupole degrees of freedom. As expected, the domain wall shows a high maximum velocity without a Walker breakdown, and the coherent precession shows a tunable frequency from sub- $\mathrm{THz}$ to THz. Our results indicate that $\mathrm{Mn}_{3} \mathrm{Sn}$ is a good candidate with many desirable properties for applications, owing to its octupole degrees of freedom.

Models. Here, we consider spin dynamics in the following Hamiltonian defined on the two-dimensional kagome lattice, which is known as a minimal model describing the Néel state of $\mathrm{Mn}_{3} \mathrm{Sn}$ [41-44],

$$
\begin{aligned}
H= & J \sum_{\langle i a, j b\rangle} \boldsymbol{S}_{i a} \cdot \boldsymbol{S}_{j b}+D \sum_{\langle i a, j b\rangle} \epsilon_{a b} \hat{\boldsymbol{z}} \cdot\left(\boldsymbol{S}_{i a} \times \boldsymbol{S}_{j b}\right) \\
& -\frac{K_{\perp}}{2} \sum_{i a}\left(\hat{\boldsymbol{K}}_{a} \cdot \boldsymbol{S}_{i a}\right)^{2},
\end{aligned}
$$



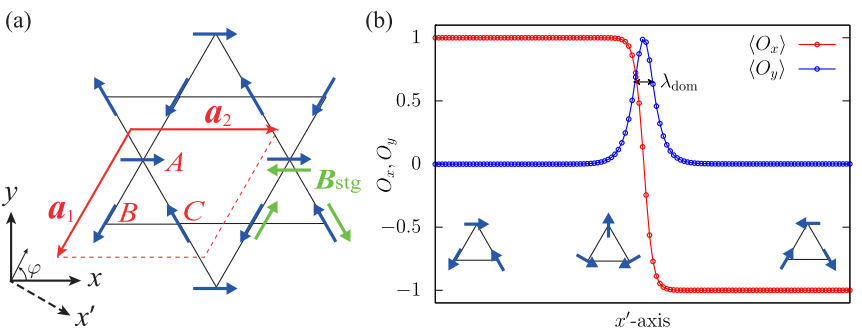

FIG. 1. (a) Spin configuration in the Néel state of $\mathrm{Mn}_{3} \mathrm{Sn}$, which is regarded as the ferroic order of the cluster octupole $O_{x}$. The nearly degenerate state corresponding to $O_{y}$ is obtained by $90^{\circ}$ rotation of each spin. (b) Schematic picture of a domain wall. From $x^{\prime}=0$ to $L, O_{x}$ changes sign from +1 to -1 , and $O_{y}$ appears when $O_{x} \simeq 0$, i.e., near the domain wall. The wall is profiled well with the two octupoles.

where the suffixes $i, j$ denote a unit cell, $a, b \in\{A, B, C\}$ denote a sublattice, and $\epsilon_{a b}$ is an antisymmetric tensor satisfying $\epsilon_{A B}=\epsilon_{B C}=\epsilon_{C A}=1$ [see Fig. 1(a)]. $J$ and $D$ represent a nearest-neighbor exchange interaction $(J>0)$ and a Dzyaloshinskii-Moriya (DM) interaction, respectively. The classical ground state of $H$ depends on the sign of $D$, and degenerate $120^{\circ}$ spin textures corresponding to the Néel states of $\mathrm{Mn}_{3} \mathrm{Sn}$ are realized when $D$ is positive. The inplane anisotropy $K_{\perp}>0$ with $\hat{\boldsymbol{K}}_{a}=\left(\cos \psi_{a}, \sin \psi_{a}, 0\right)$ and $\left(\psi_{A}, \psi_{B}, \psi_{C}\right)=\left(0, \frac{4 \pi}{3}, \frac{2 \pi}{3}\right)$ lifts the degeneracy, resulting in an $O_{x}$ octupole as the ground state $[45,48]$. Here, the spin dynamics in $\mathrm{Mn}_{3} \mathrm{Sn}$ is considered based on the LandauLifshitz-Gilbert (LLG) equations, which are formally written as

$$
\dot{\boldsymbol{S}}_{i a}=\frac{\delta H}{\hbar \delta \boldsymbol{S}_{i a}} \times \boldsymbol{S}_{i a}-\frac{\alpha}{S} \boldsymbol{S}_{i a} \times \dot{\boldsymbol{S}}_{i a}+\boldsymbol{T}_{i a}^{\mathrm{ext}},
$$

where $\boldsymbol{T}_{i a}^{\text {ext }}$ represents the torque acting on the spin $\boldsymbol{S}_{i a}$, which comes from the external magnetic field or current in this Rapid Communication. $\alpha$ denotes a Gilbert damping coefficient. In the numerical calculations, we follow previous studies and set $S=1, \alpha=0.01, K_{\perp}=0.05 J$, and $\sqrt{3} D=J$ or $J / 3$ $[37,49,50]$.

Effective theory. Although Eq. (2) with Eq. (1) can be solved numerically, in order to grasp the physics and reduce the computational cost for future applications, we then derive an effective model describing the low-energy spin dynamics in $\mathrm{Mn}_{3} \mathrm{Sn}$. When $K_{\perp}=0$, each unit cell has $D_{6 h}$ point group symmetry and the possible spin textures can be classified into its irreducible representations [33,52]. For example, the ground state shows a spin texture identified as the cluster octupole $O_{x} / O_{y}$, which belongs to $E_{1 g}$ irreducible representation,

$$
\boldsymbol{O}_{i}=\frac{1}{\sqrt{3}}\left(\overline{\boldsymbol{S}}_{i A}+R_{\frac{4 \pi}{3}} \overline{\boldsymbol{S}}_{i B}+R_{\frac{2 \pi}{3}} \overline{\boldsymbol{S}}_{i C}\right),
$$

where $\overline{\boldsymbol{S}}_{i a}=\left(S_{i a}^{x}, S_{i a}^{y}\right), \boldsymbol{O}_{i}=\left(O_{i x}, O_{i y}\right)$, and $R_{\theta}$ is the twodimensional rotation matrix. The other multipoles $m_{i \mu}(\mu=$ $1, \ldots, 7)$, corresponding to the other spin textures, are constructed as linear combinations of spins in similar ways [53]. Using these transformations, we can derive the LLG equations in the multipole representation from the original Eq. (2). An advantage in deriving such LLG comes from the fact that
TABLE I. Summary of the parameters appearing in the effective model (4). Domain wall width $\lambda_{\text {dom }}$, steady-state wall velocity $v_{\text {steady }}$, and relaxation time $\tau_{\text {relax }}$ are respectively given by $\lambda_{\text {dom }}^{2}=\kappa / \gamma$, $\hbar v_{\text {steady }}=g \mu_{B} B \lambda_{\text {dom }} / \alpha$, and $\tau_{\text {relax }}=\tau / \alpha$. Maximum wall velocity is dominated by Walker breakdown $\left(\hbar v_{\mathrm{WB}}=\lambda_{\text {dom }} K_{z} / 2\right)$ in FM and spin wave $\left(\hbar v_{\mathrm{SW}}=\sqrt{\hbar \kappa / \tau}\right)$ in $\mathrm{AFM} / \mathrm{Mn}_{3} \mathrm{Sn}$.

\begin{tabular}{lcccc}
\hline \hline Models & $\hbar \tau^{-1}$ & $\kappa / a_{\text {lat }}^{2}$ & $\gamma$ & $v_{\text {max }}$ \\
\hline FM & $K_{z}$ & $|J|$ & $K_{\perp}$ & $v_{\text {WB }}$ \\
AFM & $8|J|+K_{z}$ & $|J|$ & $K_{\perp}$ & $v_{\text {SW }}$ \\
$\mathrm{Mn}_{3}$ Sn & $2 \sqrt{3} D+6|J|$ & $(\sqrt{3} D+|J|) / 2$ & $K_{\perp}$ & $v_{\mathrm{SW}}$ \\
\hline \hline
\end{tabular}

the spin configurations corresponding to $m_{i \mu}$ have at least $\sqrt{3} D$ higher energy than $O_{x} / O_{y}$. Thus, we can systematically extract an effective model only composed of $O_{x} / O_{y}$ by integrating out the small $m_{i \mu}$ degrees of freedom. Then, the spin dynamics of the effective model can be understood in terms of two cluster octupoles.

When parametrizing $\boldsymbol{O}_{i}=\left|\boldsymbol{O}_{i}\right|\left(\cos \varphi_{i}, \sin \varphi_{i}\right)$ and taking the continuum limit, we finally obtain the following equation of motion for $\varphi\left(t, x^{\prime}\right)$,

$$
\tau \hbar \ddot{\varphi}+\alpha \hbar \dot{\varphi}-\kappa \partial^{2} \varphi+\frac{\gamma}{2} \sin (2 \varphi)=T_{\mathrm{ext}},
$$

where the parameters are given by $\hbar \tau^{-1}=2 \sqrt{3} D+6|J|, \kappa=$ $a_{\text {lat }}^{2}(\sqrt{3} D+|J|) / 2$, and $\gamma=K_{\perp}$ [54]. Here, $a_{\text {lat }}$ is the distance between the nearest-neighbor spins and we have set $S=1$. We have also assumed that $\varphi$ is uniform along the $\boldsymbol{a}_{1}$ direction [for the definitions of $x^{\prime}$ and $\boldsymbol{a}_{1}$, see Fig. 1(a)]. The force term $T_{\text {ext }}$ generally depends on the external torque $\boldsymbol{T}_{i a}^{\text {ext }}$. Note that Eq. (4) is defined in the continuum space and can be scaled by renormalizing the stiffness parameter $\kappa$, and thus would be useful in micromagnetic simulations.

To derive Eq. (4), we have assumed (1) the cluster multipoles are slowly varying with respect to $a_{\text {lat }}$, (2) the cluster octupoles are energetically stable, and thus $\left|O_{i x}\right|,\left|O_{i y}\right| \gg$ $\left|m_{i \mu}\right|$, and (3) $1 / S$ and $K_{\perp} / J$ are also small. The minor multipole contributions are included within the lowest order of these small parameters. (For details, see the Supplemental Material [53].) The above assumptions are plausible whenever the system possesses a robust low-energy collective motion. Thus, our approach would be applicable to a wide class of magnets unless they host a number of nearly degenerate spin configurations at low energy. Indeed, Eq. (4), the sine-Gordon form, is completely the same as in collinear FM and AFM. For example, let us consider the following Hamiltonian on the two-dimensional square lattice,

$$
H=-J \sum_{\langle i, j\rangle} S_{i} \cdot S_{j}+\frac{1}{2} \sum_{i}\left[K_{z}\left(S_{i}^{z}\right)^{2}-K_{\perp}\left(S_{i}^{x}\right)^{2}\right],
$$

where $K_{z}, K_{\perp}>0$, and $J>0(J<0)$ for the collinear FM (AFM). Using this Hamiltonian with $|J|>K_{z} \gg K_{\perp}, \varphi\left(t, x^{\prime}\right)$ appearing in Eq. (4) respectively corresponds to the in-plane angle of the spin in FM and that of the Néel vector, defined as the difference between the spins on two sublattices, in AFM. In the same manner, we can derive the effective model and identify the parameters $\tau, \kappa$, and $\gamma$ for FM and AFM, which are summarized in Table I. The typical timescale of AFM and 


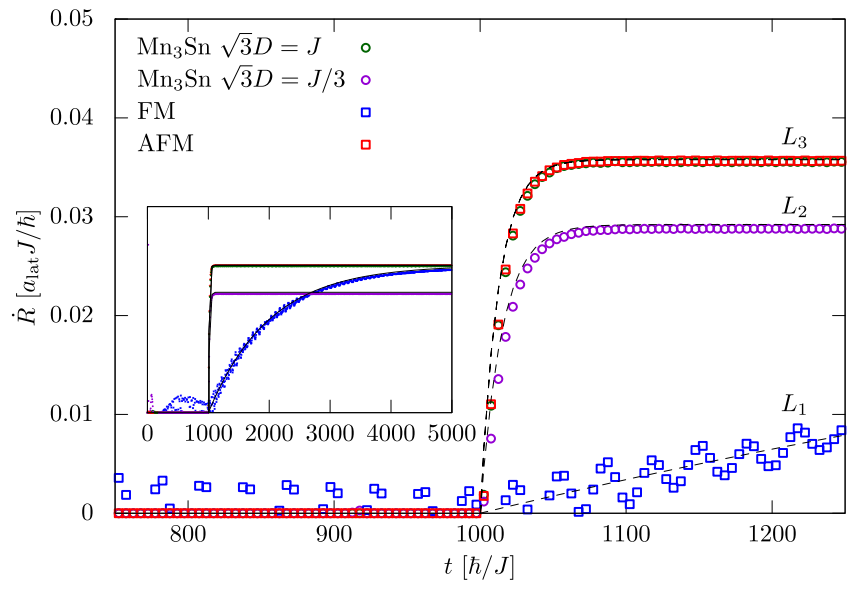

FIG. 2. Domain wall velocity $\dot{R}(t)$. Staggered magnetic field, which is set to be $g \mu_{B} B_{\text {stg }}=8 \times 10^{-5} \mathrm{~J}$, is applied for $t>1000 \hbar / J$. The open squares represent the results for collinear FM (blue) and AFM (red). The open circles represent those for $\mathrm{Mn}_{3} \mathrm{Sn}$ with $\sqrt{3} D=J$ (green) and $\sqrt{3} D=J / 3$ (purple). The dashed black lines $L_{1}, L_{2}$, and $L_{3}$ indicate analytic solutions given in Eq. (6) for FM, $\mathrm{Mn}_{3} \operatorname{Sn}(\sqrt{3} D=J / 3)$, and $\mathrm{AFM} / \mathrm{Mn}_{3} \operatorname{Sn}(\sqrt{3} D=J)$, respectively.

$\mathrm{Mn}_{3} \mathrm{Sn}$ is given by $\mathcal{O}\left(\hbar J^{-1}\right)$, which is usually much faster than that of FM of $\mathcal{O}\left(\hbar K_{z}^{-1}\right)$. As will be seen later, this results in a short time relaxation of the domain wall motion as well as a $\mathrm{THz}$ coherent precession. Another notable point is that when $J$ and $D$ satisfy $\sqrt{3} D=J$, all parameters in Eq. (4) are the same in between collinear AFM and $\mathrm{Mn}_{3} \mathrm{Sn}$ up to the first order of $J / K_{z}$. Thus, we can expect that the spin dynamics of collinear $\mathrm{AFM}$ and $\mathrm{Mn}_{3} \mathrm{Sn}$ are essentially the same in this limit.

Domain wall motion. In the following, we will see the validity of our effective model to calculate the domain wall dynamics. It should be noted that, similar to collinear AFM, the torque coming from the uniform magnetic field cancels out in each unit cell and does not drive the domain wall. Here, we simply apply the staggered magnetic field by adding $H_{\mathrm{ext}}=-g \mu_{B} B_{\mathrm{stg}} \sum_{i a}\left(\hat{\boldsymbol{K}}_{a} \cdot \boldsymbol{S}_{i a}\right)$ to $H$, which results in an effective torque as $T_{\text {ext }}=-g \mu_{B} B_{\text {stg }} \sin \varphi[53,55]$. To obtain a domain wall solution, we take the boundary condition such that $\varphi(t, 0)=0$ and $\varphi(t, L)=\pi$ [see Fig. 1(b)]. Assuming an equilibrium solution with the profile $\cos \varphi\left(t, x^{\prime}\right)=$ $\tanh \left[\left(x^{\prime}-R\right) / \lambda_{\mathrm{dom}}\right]$ and resubstituting it to the action by interpreting the constant of the integration $R$ as the timedependent variable describing the domain wall center, we obtain

$$
\dot{R}(t)=v_{\text {steady }}\left(1-e^{-t / \tau_{\text {relax }}}\right),
$$

which satisfies $\dot{R}(0)=0 . \hbar v_{\text {steady }}=g \mu_{B} B_{\text {stag }} \lambda_{\text {dom }} / \alpha$ is the domain wall velocity in the steady state and $\tau_{\text {relax }}=\tau / \alpha$ is the typical timescale to relax into it.

Figure 2 shows numerical results for the domain wall velocity obtained by solving Eq. (2) [53] and the analytic solutions given by Eq. (6). From the figure, we can see that the analytic solutions agree well with the numerical results except for the small oscillating behavior in FM [56]. As expected, the relaxation time to reach $v_{\text {steady }}$ is much faster in $\mathrm{AFM} / \mathrm{Mn}_{3} \mathrm{Sn}$ than in $\mathrm{FM}$, and the behavior of $\mathrm{Mn}_{3} \mathrm{Sn}$ with $\sqrt{3} D=J$ is almost the same as AFM. Figure 2 clearly shows that our

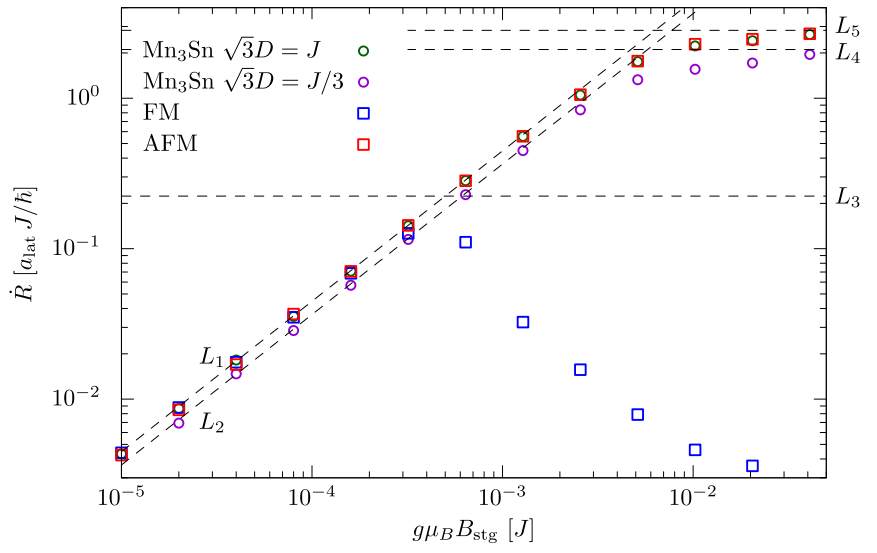

FIG. 3. Steady-state domain wall velocity $\dot{R}$ as a function of the staggered magnetic field. The open symbols are defined in the same way as in Fig. 2. The lines $L_{1}$ and $L_{2}$ show $v_{\text {steady }}$ corresponding to $\mathrm{FM} / \mathrm{AFM} / \mathrm{Mn}_{3} \operatorname{Sn}(\sqrt{3} D=J)$ and $\mathrm{Mn}_{3} \operatorname{Sn}(\sqrt{3}=J / 3)$, respectively. $L_{3}, L_{4}$, and $L_{5}$ indicate the saturation values, i.e., $v_{\mathrm{WB}}$ for FM, $v_{\mathrm{SW}}$ for $\mathrm{Mn}_{3} \operatorname{Sn}(\sqrt{3} D=J / 3)$, and $\mathrm{AFM} / \mathrm{Mn}_{3} \operatorname{Sn}(\sqrt{3} D=J)$, respectively.

effective model correctly represents the original model not only in FM/AFM but also in $\mathrm{Mn}_{3} \mathrm{Sn}$ regardless of the value of $D$.

In Fig. 3, we show the field strength dependence of the steady-state velocity. At a low-field region, the domain wall velocity is proportional to $B_{\text {stg }}$ and is almost on the lines $v_{\text {steady }}=g \mu_{B} B_{\text {stg }} \lambda_{\text {dom }} / \alpha$ in all cases. However, at a high-field region, the behavior in FM is different from the other cases, because of the presence (absence) of the Walker breakdown in FM (AFM/ $\left./ \mathrm{Mn}_{3} \mathrm{Sn}\right)$. The absence of the Walker breakdown in AFM can be understood as follows: The trigger of the Walker breakdown is the tilt of spins to the out-of-plane direction due to the torque, which arranges the spins to the same direction. However, in contrast to FM, such a spin configuration losses the exchange energy of order $\mathcal{O}(J)$, and thus does not occur unless $g \mu_{B} B_{\text {stg }}$ exceeds $J$ [9-11]. In $\mathrm{Mn}_{3} \mathrm{Sn}$, the situation is the same as AFM and the Walker breakdown does not occur. Thus, the saturation velocity in AFM/ $\mathrm{Mn}_{3} \mathrm{Sn}$ is simply determined by the Lorentz boost of the equilibrium solution and given by the spin-wave velocity $\hbar v_{\mathrm{SW}}=\sqrt{\kappa / \tau}$ while that in FM is given by the Walker breakdown $\hbar v_{\mathrm{WB}}=\lambda_{\text {dom }} K_{z} / 2$, which are indicated in Fig. 3. Using the parameters $2 a_{\text {lat }}=$ $5.4 \AA, J=2.8 \mathrm{meV}, D=0.64 \mathrm{meV}$, and $S=3 / 2[37,57]$, we estimate $v_{\mathrm{SW}} \simeq 2 \mathrm{~km} / \mathrm{s}$ in $\mathrm{Mn}_{3} \mathrm{Sn}$, which is slightly smaller than the collinear AFM such as $36 \mathrm{~km} / \mathrm{s}$ of dielectric $\mathrm{NiO}$ [58] and $90 \mathrm{~km} / \mathrm{s}$ of $\mathrm{KFeS}_{2}$ [59], but still faster than the highest record in FMs of $400 \mathrm{~m} / \mathrm{s}$ [60].

Coherent precession of spins. Finally, we focus on the steady precession motion allowed in $\mathrm{Mn}_{3} \mathrm{Sn}$, which may be the source of a coherent $\mathrm{THz}$ signal. Here, we consider the system that contains a $\mathrm{Mn}_{3} \mathrm{Sn}$ thin film sandwiched by two conventional FMs along the $z$ direction [38]. When the spin accumulation polarizing along $\zeta$ exists at the interface, the torque expressed by the following form acts on the spin $\boldsymbol{S}_{i a}$,

$$
\hbar \boldsymbol{T}_{i a}^{\mathrm{ext}}=\tau_{F} \boldsymbol{S}_{i a} \times \zeta+\frac{\tau_{D}}{S} \boldsymbol{S}_{i a} \times\left(\zeta \times \boldsymbol{S}_{i a}\right)
$$


(a)
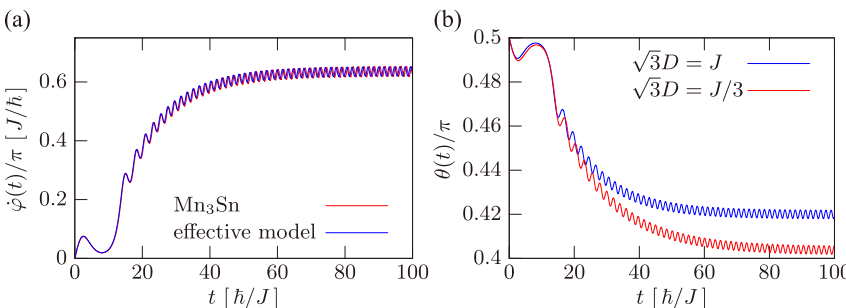

(c)

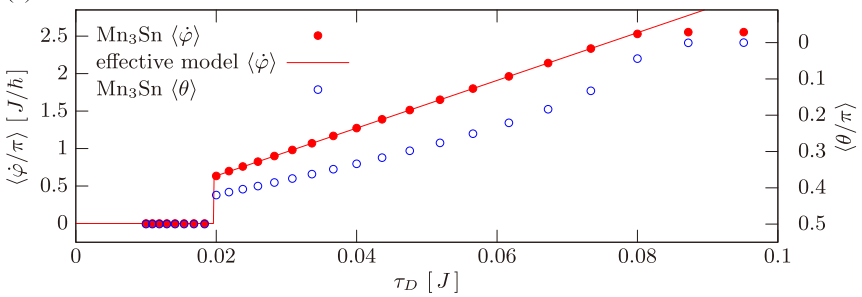

FIG. 4. (a) Time evolution of space-averaged $\dot{\varphi}(t)$ when $\tau_{D}=$ $0.02 J$. Red and blue lines respectively show the results by solving Eqs. (2) and (4) numerically. (b) Time evolution of the polar angle $\theta(t)$ of each spin obtained by solving Eq. (2). (c) Time- and spaceaveraged $\langle\dot{\varphi}\rangle$ and $\langle\theta\rangle$ in the steady state. The slope of the red line is given by $\hbar\langle\dot{\varphi}\rangle=\tau_{D} / \alpha$.

where the first term, called a fieldlike torque, represents the exchange interaction between the spins, while the second term, called a dampinglike torque, comes from the conservation of the spin angular momentum through the dissipation $[61,62]$. Although both $\tau_{F}$ and $\tau_{D}$ are proportional to the injected spin current [63], the first term does not drive the steady precession and we only take into account the second term in the following. Also, we set $\zeta=(0,0,1)$, resulting in the constant force $T_{\text {ext }}=\tau_{D}$ in the effective model [53], and impose the periodic boundary condition on the system. In the effective model, we can simply neglect the $x^{\prime}$ dependence of $\varphi\left(t, x^{\prime}\right)$, and then the model coincides with the second Josephson equation under a current bias [18,64].

Figure 4(a) shows the space-averaged $\dot{\varphi}(t)$ obtained by solving the original LLG (2) with the torque (7) and the effective model (4) with $T_{\text {ext }}=\tau_{D}$, where $\tau_{D}=0.02 \mathrm{~J}$. We can see that the coherent precession of octupoles is really realized and it does not decay with time. The agreement between the original and the effective models is very good. The mechanism of such a steady precession can be understood in the same way as in FM: The dissipation of the spin angular momentum through the Gilbert damping exactly compensates the provided one through the dampinglike torque, namely, the dissipation of the accumulated spins. The velocity of the precession in $\mathrm{Mn}_{3} \mathrm{Sn}$, however, is much higher than the FM because the dampinglike torque rather competes with the exchange $J$ and the DM interaction $D$ [Fig. 4(b)] than the external field $B_{z}$ or the anisotropy $K_{z}$ in the case of FM. That implies that the precession frequency reaches $\mathcal{O}(J / \hbar)$ in the limit that all spins are along the $z$ direction.

It is worth noting that the steady state $\dot{\varphi}(t)$ is not constant with time and oscillates as seen in Fig. 4(a). This comes from the out-of-plane anisotropy $K_{z}$ in the case of collinear AFM [18], and the DM interaction plays a similar role in $\mathrm{Mn}_{3} \mathrm{Sn}$. In collinear AFM, only a small oscillation of $\dot{\varphi}(t)$ is detectable through the inverse spin-Hall effects while we can directly detect the whole octupole precession motion such as through the magneto-optical Kerr effect [46] and an oscillation of the Hall voltage [53]. This is a clear advantage of $\mathrm{Mn}_{3} \mathrm{Sn}$ over collinear AFM.

Figure 4(c) shows space- and time-averaged $\langle\dot{\varphi}\rangle$ and $\langle\theta\rangle$ (the polar angle of the spins) in the steady state. $\langle\dot{\varphi}\rangle$ of the effective model is simply given by $\hbar\langle\dot{\varphi}\rangle=\tau_{D} / \alpha$, and again agrees well with the LLG calculations. The maximum frequency $f_{\max }$ of the precession is achieved where all spins are along the $z$ direction and is estimated as $f_{\max }=$ $(2 \sqrt{3} D+6 J) / h \simeq 7.2 \mathrm{THz}[65]$, which is comparable to the magnon frequency of $\mathrm{KFeS}_{2}$ [59]. On the other hand, owing to the extremely small in-plane anisotropy of $\mathrm{Mn}_{3} \mathrm{Sn}$, the threshold frequency $f_{\text {thr }} \sim \mathcal{O}\left(K_{\perp} / \alpha h\right)$ is about $10 \mathrm{GHz}$ $[51,66]$. Thus, the frequency in the range of three orders of magnitude may be available in $\mathrm{Mn}_{3} \mathrm{Sn}$.

Conclusion. In this Rapid Communication, we develop a method to obtain a low-energy effective model of noncollinear AFM based on the cluster multipole theory and apply it to a simple model of $\mathrm{Mn}_{3} \mathrm{Sn}$. A comparison between the original and effective models shows good agreement both in the domain wall dynamics and in the coherent steady precession of spins. This means that the low-energy dynamics of $\mathrm{Mn}_{3} \mathrm{Sn}$ is almost dominated by the octupole degrees of freedom and we do not have to trace that of each spin, which enables us to reduce the computational cost. Our results show that the octupole dynamics in $\mathrm{Mn}_{3} \mathrm{Sn}$ is almost the same as that of the Néel vector in collinear AFM, which indicates that $\mathrm{Mn}_{3} \mathrm{Sn}$ possesses advantages of AFM as well as of FM. Thus, $\mathrm{Mn}_{3} \mathrm{Sn}$ would be a promising candidate for future applications in multipole-based electronics.

Acknowledgments. We are grateful to W. Koshibae, S. Miwa, Y. Otani, S. Nakatsuji, K. Yakushiji, and S. Yuasa for many valuable discussions. This work was supported by a Grant-in-Aid for Scientific Research (No. 16H06345) from Ministry of Education, Culture, Sports, Science and Technology, Japan and CREST (Grants No. JPMJCR15Q5 and No. JPMJCR18T3), the Japan Science and Technology Agency.
[1] E. V. Gomonay and V. M. Loktev, Low Temp. Phys. 40, 17 (2014).

[2] T. Jungwirth, X. Marti, P. Wadley, and J. Wunderlich, Nat. Nanotechnol. 11, 231 (2016).

[3] O. Gomonay, T. Jungwirth, and J. Sinova, Phys. Status Solidi RRL 11, 1700022 (2017).

[4] V. Baltz, A. Manchon, M. Tsoi, T. Moriyama, T. Ono, and Y. Tserkovnyak, Rev. Mod. Phys. 90, 015005 (2018).
[5] O. Gomonay, V. Baltz, A. Brataas, and Y. Tserkovnyak, Nat. Phys. 14, 213 (2018).

[6] A. Manchon, I. M. Miron, T. Jungwirth, J. Sinova, J. Železný, A. Thiaville, K. Garello, and P. Gambardella, Rev. Mod. Phys. 91, 035004 (2019).

[7] J. Železný, P. Wadley, K. Olejník, A. Hoffmann, and H. Ohno, Nat. Phys. 14, 220 (2018).

[8] R. Cheng and Q. Niu, Phys. Rev. B 89, 081105(R) (2014). 
[9] O. Gomonay, T. Jungwirth, and J. Sinova, Phys. Rev. Lett. 117, 017202 (2016).

[10] T. Shiino, S.-H. Oh, P. M. Haney, S.-W. Lee, G. Go, B.-G. Park, and K.-J. Lee, Phys. Rev. Lett. 117, 087203 (2016).

[11] S. Selzer, U. Atxitia, U. Ritzmann, D. Hinzke, and U. Nowak, Phys. Rev. Lett. 117, 107201 (2016).

[12] A. V. Kimel, A. Kirilyuk, A. Tsvetkov, R. V. Pisarev, and T. Rasing, Nature (London) 429, 850 (2004).

[13] A. V. Kimel, B. A. Ivanov, R. V. Pisarev, P. A. Usachev, A. Kirilyuk, and Th. Rasing, Nat. Phys. 5, 727 (2009).

[14] T. Satoh, S.-J. Cho, R. Iida, T. Shimura, K. Kuroda, H. Ueda, Y. Ueda, B. A. Ivanov, F. Nori, and M. Fiebig, Phys. Rev. Lett. 105, 077402 (2010).

[15] J. Nishitani, T. Nagashima, and M. Hangyo, Phys. Rev. B 85, 174439 (2012).

[16] R. Cheng, J.-G. Zhu, and D. Xiao, Phys. Rev. Lett. 117, 097202 (2016).

[17] R. Cheng, D. Xiao, and A. Brataas, Phys. Rev. Lett. 116, 207603 (2016).

[18] R. Khymyn, I. Lisenkov, V. Tiberkevich, B. A. Ivanov, and A. Slavin, Sci. Rep. 7, 43705 (2017).

[19] O. R. Sulymenko, O. V. Prokopenko, V. S. Tiberkevich, A. N. Slavin, B. A. Ivanov, and R. S. Khymyn, Phys. Rev. Appl. 8, 064007 (2017).

[20] M. Binder, A. Weber, O. Mosendz, G. Woltersdorf, M. Izquierdo, I. Neudecker, J. R. Dahn, T. D. Hatchard, J.-U. Thiele, C. H. Back, and M. R. Scheinfein, Phys. Rev. B 74, 134404 (2006).

[21] C. D. Stanciu, A. V. Kimel, F. Hansteen, A. Tsukamoto, A. Itoh, A. Kiriliyuk, and T. Rasing, Phys. Rev. B 73, 220402(R) (2006).

[22] Y. Ohnuma, H. Adachi, E. Saitoh, and S. Maekawa, Phys. Rev. B 87, 014423 (2013).

[23] J. Finley and L. Q. Liu, Phys. Rev. Appl. 6, 054001 (2016).

[24] R. Mishra, J. Yu, X. Qiu, M. Motapothula, T. Venkatesan, and H. Yang, Phys. Rev. Lett. 118, 167201 (2017).

[25] K.-J. Kim, S. K. Kim, Y. Hirata, S.-H. Oh, T. Tono, D.-H. Kim, T. Okuno, W. S. Ham, S. Kim, G. Go, Y. Tserkovnyak, A. Tsukamoto, T. Moriyama, K.-J. Lee, and T. Ono, Nat. Mater. 16, 1187 (2017).

[26] K. Kuroda, T. Tomita, M.-T. Suzuki, C. Bareille, A. A. Nugroho, P. Goswami, M. Ochi, M. Ikhlas, M. Nakayama, S. Akebi, R. Noguchi, R. Ishii, N. Inami, K. Ono, H. Kumigashira, A. Varykhalov, T. Muro, T. Koretsune, R. Arita, S. Shin, T. Kondo, and S. Nakatsuji, Nat. Mater. 16, 1090 (2017).

[27] J. Kübler and C. Felser, Europhys. Lett. 108, 67001 (2014).

[28] S. Nakatsuji, N. Kiyohara, and T. Higo, Nature (London) 527, 212 (2015).

[29] N. Kiyohara, T. Tomita, and S. Nakatsuji, Phys. Rev. Appl. 5, 064009 (2016).

[30] H. Yang, Y. Sun, Y. Zhang, W.-J. Shi, S. S. P. Parkin, and B. Yan, New J. Phys. 19, 015008 (2017).

[31] M. Ikhlas, T. Tomita, T. Koretsune, M.-T. Suzuki, D. NishioHamane, R. Arita, Y. Otani, and S. Nakatsuji, Nat. Phys. 13, 1085 (2017).

[32] X. Li, L. Xu, L. Ding, J. Wang, M. Shen, X. Lu, Z. Zhu, and K. Behnia, Phys. Rev. Lett. 119, 056601 (2017).

[33] M.-T. Suzuki, T. Koretsune, M. Ochi, and R. Arita, Phys. Rev. B 95, 094406 (2017).
[34] A. K. Nayak, J. E. Fischer, Y. Sun, B. Yan, J. Karel, A. C. Komarek, C. Shekhar, N. Kumar, W. Schnelle, J. Kübler, C. Felser, and S. S. P. Parkin, Sci. Adv. 2, e1501870 (2016).

[35] H. V. Gomonay, R. V. Kunitsyn, and V. M. Loktev, Phys. Rev. B 85, 134446 (2012).

[36] K. Prakhya, A. Popescu, and P. M. Haney, Phys. Rev. B 89, 054421 (2014).

[37] J. Liu and L. Balents, Phys. Rev. Lett. 119, 087202 (2017).

[38] H. Fujita, Phys. Status Solidi RRL 11, 1600360 (2017).

[39] M.-T. Suzuki, H. Ikeda, and P. M. Oppeneer, J. Phys. Soc. Jpn. 87, 041008 (2018).

[40] M.-T. Suzuki, T. Nomoto, R. Arita, Y. Yanagi, S. Hayami, and H. Kusunose, Phys. Rev. B 99, 174407 (2019).

[41] S. Tomiyoshi and Y. Yamaguchi, J. Phys. Soc. Jpn. 51, 2478 (1982).

[42] T. Nagamiya, S. Tomiyoshi, and Y. Yamaguchi, Solid State Commun. 42, 385 (1982).

[43] J. W. Cable, N. Wakabayashi, and P. Radhakrishna, Phys. Rev. B 48, 6159 (1993).

[44] P. Park, J. Oh, K. Uhlîrová, J. Jackson, A. Deák, L. Szunyogh, K. H. Lee, H. Cho, H.-L. Kim, H. C. Walker, D. Adroja, V. Sechovský, and J.-G. Park, npj Quantum Mater. 3, 63 (2018).

[45] In Refs. [37,38], a different type of single-ion (locally twofold symmetric) anisotropy is considered, which preserves global sixfold symmetry and reproduces small canting in $\mathrm{Mn}_{3} \mathrm{Sn}$. In this Rapid Communication, however, we do not take it into account to focus on the spin dynamics of noncollinear AFM without a net magnetization. The twofold anisotropy in the present study is also based on the experimental realization of $180^{\circ}$ domains as shown in Fig. 1(b) $[46,47]$.

[46] T. Higo, H. Man, D. B. Gopman, L. Wu, T. Koretsune, O. M. J. van't Erve, Y. P. Kabanov, D. Rees, Y. Li, M.-T. Suzuki, S. Patankar, M. Ikhlas, C. L. Chien, R. Arita, R. D. Shull, J. Orenstein, and S. Nakatsuji, Nat. Photonics 12, 73 (2018).

[47] X. Li, C. Collignon, L. Xu, H. Zuo, A. Cavanna, U. Gennser, D. Mailly, B. Fauqué, L. Balents, Z. Zhu, and K. Behnia, Nat. Commun. 10, 3021 (2019).

[48] This corresponds to the spin texture of $\mathrm{Mn}_{3} \mathrm{Sn}$ realized in the temperature range between the Néel temperature and $\sim 50 \mathrm{~K}$.

[49] A. Brataas, Y. Tserkovnyak, G. E. W. Bauer, and P. J. Kelly, in Spin Current, edited by S. Maekawa, S. O. Valenzuela, E. Saitoh, and T. Kimura (Oxford University Press, Oxford, UK, 2012).

[50] As mentioned in Ref. [45], our in-plane anisotropy $K_{\perp}$ should be regarded as an effective one to reproduce the $180^{\circ}$ domains. Although the experimental value of $K_{\perp}$ is much smaller than $0.05 \mathrm{~J}$ [51], the size of $K_{\perp}$ is irrelevant to the following discussion.

[51] S. Miwa, S. Iihama, T. Nomoto, T. Tomita, T. Higo, M. Ikhlas, Y. Otani, S. Mizukami, R. Arita, and S. Nakatsuji (unpublished).

[52] In actual $\mathrm{Mn}_{3} \mathrm{Sn}$, each unit cell has only $D_{3 d}$ symmetry due to the presence of interlayer couplings. Here, we follow the notation defined in our simplified model.

[53] See Supplemental Material at http://link.aps.org/supplemental/ 10.1103/PhysRevResearch.2.012045 for details of the derivation of the effective model, numerical simulation and oscillation of the Hall voltage. 
[54] The mass term with $\gamma \propto K_{\perp}^{3}$ and sixfold symmetry in Ref. [37] is not predicted in our model. This comes from the difference of the anisotropy terms in the original models (see Ref. [45]).

[55] At low energy, the staggered field has almost the same effect as the dampinglike torque introduced by Eq. (7).

[56] This oscillation originates from a long lifetime precession mode in FM, which is irrelevant to our arguments.

[57] If we take into account the out-of-plane anisotropy $K_{z}$ in $\mathrm{Mn}_{3} \mathrm{Sn}$, we obtain a higher maximum velocity since $\hbar \tau^{-1}$ changes from $2 \sqrt{3} D+6 J$ to $2 \sqrt{3} D+6 J+K_{z}$ [53].

[58] M. T. Hutchings and E. J. Samuelsen, Phys. Rev. B 6, 3447 (1972).

[59] D. Welz, M. Kohgi, Y. Endoh, M. Nishi, and M. Arai, Phys. Rev. B 45, 12319 (1992).
[60] I. M. Miron, T. Moore, H. Szambolics, L. D. Buda-Prejbeanu, S. Auffret, B. Rodmacq, S. Pizzini, J. Vogel, M. Bonfim, A. Schuhl, and G. Gaudin, Nat. Mater. 10, 419 (2011).

[61] L. Berger, Phys. Rev. B 54, 9353 (1996).

[62] J. C. Slonczewski, J. Magn. Magn. Mater. 159, L1 (1996).

[63] Using current density $j, \tau_{D}$ is expressed as $\tau_{D}=\frac{g \mu_{B} \hbar P}{2 e M_{s} d} j$, where $M_{s}, P$, and $d$ are the saturation magnetization, spin polarization, and thickness of the film, respectively.

[64] W. C. Stewart, Appl. Phys. Lett. 12, 277 (1968).

[65] Again, the out-of-plane anisotropy $K_{z}$ enhances $f_{\max }$ due to the change of $\hbar \tau^{-1}$ [57]

[66] The corresponding threshold current density $j_{\text {thr }}$ is about $10^{10} \mathrm{~A} \mathrm{~m}^{-2}$ obtained by a simple estimation with $P=1$ and $d=1 \mathrm{~nm}$. 